\title{
Detection of severe acute respiratory coronavirus virus 2 (SARS-CoV-2) in outpatients: A multicenter comparison of self-collected saline gargle, oral swab, and combined oral-anterior nasal swab to a provider collected nasopharyngeal swab
}

\author{
Christopher E. Kandel ${ }^{1}$ (1), Matthew Young ${ }^{2}$, Mihaela Anca Serbanescu ${ }^{3}$, Jeff E. Powis ${ }^{2}$, David Bulir,5, James Callahan ${ }^{2}$, \\ Kevin Katz ${ }^{6,7,8}$, Janine McCready ${ }^{2}$, Hilary Racher ${ }^{3,6}$, Elena Sheldrake ${ }^{7}$, Dorothy Quon ${ }^{2}$, Omid Kyle Vojdani², \\ Allison McGeer ${ }^{9}$, Lee W. Goneau ${ }^{3,6, a}$ and Christie Vermeiren ${ }^{7, a}$ \\ ${ }^{1}$ Faculty of Medicine, University of Toronto, Toronto, Ontario, Canada, ${ }^{2}$ Michael Garron Hospital, University of Toronto, Toronto, Ontario, Canada, ${ }^{3}$ Dynacare \\ Laboratory, Brampton, Canada, ${ }^{4}$ Department of Pathology and Molecular Medicine, McMaster University, Hamilton, Ontario, Canada, ${ }^{5}$ Research Institute of \\ St Joe's Hamilton, Hamilton, Ontario, Canada, ${ }^{6}$ Department of Laboratory Medicine, University of Toronto, Toronto, Ontario, Canada, ${ }^{7}$ Shared Hospital \\ Laboratory, Toronto, Ontario, Canada, ${ }^{8}$ North York General Hospital, Toronto, Ontario, Canada and ${ }^{9}$ Mount Sinai Hospital, Toronto, Ontario, Canada
}

\begin{abstract}
Background: Widespread testing for severe acute respiratory coronavirus virus 2 (SARS-CoV-2) is necessary to curb the spread of coronavirus disease 2019 (COVID-19), but testing is undermined when the only option is a nasopharyngeal swab. Self-collected swab techniques can overcome many of the disadvantages of a nasopharyngeal swab, but they require evaluation.

Methods: Three self-collected non-nasopharyngeal swab techniques (saline gargle, oral swab and combined oral-anterior nasal swab) were compared to a nasopharyngeal swab for SARS-CoV-2 detection at multiple COVID-19 assessment centers in Toronto, Canada. The performance characteristics of each test were assessed.

Results: The adjusted sensitivity of the saline gargle was 0.90 (95\% CI 0.86-0.94), the oral swab was 0.82 (95\% CI, 0.72-0.89) and the combined oral-anterior nasal swab was 0.87 (95\% CI, 0.77-0.93) compared to a nasopharyngeal swab, which demonstrated a sensitivity of $\sim 90 \%$ when all positive tests were the reference standard. The median cycle threshold values for the SARS-CoV-2 E-gene for concordant and discordant saline gargle specimens were 17 and $31(P<.001)$, for the oral swabs these values were 17 and $28(P<.001)$, and for oral-anterior nasal swabs these values were 18 and $31(P=.007)$.

Conclusions: Self-collected saline gargle and an oral-anterior nasal swab have a similar sensitivity to a nasopharyngeal swab for the detection of SARS-CoV-2. These alternative collection techniques are cheap and can eliminate barriers to testing, particularly in underserved populations.
\end{abstract}

(Received 23 November 2020; accepted 30 December 2020; electronically published 13 January 2021)

Severe acute respiratory syndrome coronavirus 2 (SARS-CoV-2), the virus responsible coronavirus disease 2019 (COVID-19), continues to be a worldwide health crisis. The test, treat, and isolate strategy that has successfully mitigated spread is underpinned by widespread testing. ${ }^{1}$ Currently, the most widely used diagnostic

Author for correspondence: Christopher Kandel, E-mail: christopher.kandel@mail. utoronto.ca

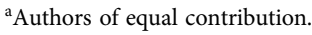

Cite this article: Kandel CE, et al. (2021). Detection of severe acute respiratory coronavirus virus 2 (SARS-CoV-2) in outpatients: A multicenter comparison of selfcollected saline gargle, oral swab, and combined oral-anterior nasal swab to a provider collected nasopharyngeal swab. Infection Control \& Hospital Epidemiology, 42: 1340-1344, https://doi.org/10.1017/ice.2021.2 test is real-time reverse transcription polymerase chain reaction (rRT- PCR) on specimens collected from the nasopharynx. This test is uncomfortable and requires travel to a facility where a trained healthcare professional wearing personal protective equipment collects the specimen. ${ }^{2}$ Self-collected specimens can overcome these and other barriers that may limit testing, particularly in disadvantaged groups that have been disproportionately affected by COVID-19. ${ }^{3}$

Of the nasopharyngeal swab-independent techniques evaluated, saliva has been the most widely assessed, with sensitivity ranging from $69.2 \%$ to $97.6 \%{ }^{4}$ The main drawbacks of using saliva are the inability of some individuals to produce sufficient quantity of saliva for testing, the challenge of processing specimens in

(c) The Author(s), 2021. Published by Cambridge University Press on behalf of The Society for Healthcare Epidemiology of America. This is an Open Access article, distributed under the terms of the Creative Commons Attribution licence (http://creativecommons.org/licenses/by/4.0/), which permits unrestricted re-use, distribution, and reproduction in any medium, provided the original work is properly cited. 
automated systems designed to receive standard swab tubes, the requirement for heat inactivation, and the need for repeat testing of invalid specimens. ${ }^{5}$ These issues may account for some of the variability in performance characteristics observed. Saline gargle and self-collected non-nasopharyngeal swabs can overcome these limitations, but these have not been assessed in outpatients. ${ }^{6}$

We assessed the performance characteristics of self-collected saline gargle, oral swab, and combined oral-anterior nasal swab for the detection of SARS-CoV-2 in 3 outpatient testing centers.

\section{Methods}

\section{Study population and self-collected specimens}

The study population was consecutive individuals presenting to 3 assessment centers in Toronto, Ontario, who had a nasopharyngeal swab obtained for SARS-CoV-2 testing. During 3 separate study periods, a self-collected paired non-nasopharyngeal specimen was requested: saline gargle from August 28 to September 25, 2020; oral swab from September 29 to October 3, 2020; and a combined oral-anterior nasal swab from October 14 to 17, 2020. For the saline gargle, individuals performed a swish and gargle 3 times with $3 \mathrm{~mL}$ of $0.9 \%$ normal saline. For the oral swab, individuals self-swabbed the back of their tongue, buccal mucosa, then coat the swab in saliva. For the combined oral-anterior nasal swab, each individual self-swabbed the back of their tongue, buccal mucosa, and the anterior aspect of both nares (for complete instructions see the Supplementary Material online). The swab used for the latter 2 techniques was the Miraclean Technology disposable flocked nasal, oral, and throat swab. All specimens were stored at $4^{\circ} \mathrm{C}$ until testing. This study was approved by the Michael Garron Hospital Research Ethics Board with implied consent for the voluntary selfcollection of a second test after the study rationale was explained.

\section{Study design}

Once the nasopharyngeal swab results were reported, all non-nasopharyngeal specimens available from individuals positive for SARS-CoV-2 and a randomly selected sample from individuals who tested negative on the same day (ratio of negative to positive 7-10 to 1) were retrieved for testing. Clinical information (age, sex, and time from symptom onset) for those who were positive by any test was abstracted by chart review from assessments performed by infectious diseases physicians.

\section{SARS-COV-2 RNA detection}

Nasopharyngeal, oral, and the combined oral-anterior nasal swabs were tested at Shared Hospital Laboratory (Toronto, ON). All swab samples were placed into a guanidine thiocyanate-based transport medium (McMaster Molecular Medium, Bay Area Health Trustee Corporation, Hamilton, Ontario). A $160-\mu \mathrm{L}$ aliquot was extracted on the MGISP-960 automated workstation using the MGI Easy Magnetic Beads Virus DNA/RNA Extraction Kit (MGI Technologies, Shenzhen, China). Detection of SARS-CoV-2 Egene and 5'-UTR and the internal control (RNase P), was performed using the Luna Universal Probe One-Step RT-qPCR kit (New England Biolabs, Whitby, Ontario) on the CFX96 Touch Real-time PCR detection system (BioRad, Mississauga, Ontario) as previously described. ${ }^{7}$ A result is considered positive when the cycle threshold $(\mathrm{Ct})$ is below 38 for both targets.

Saline gargle samples were tested at Dynacare Laboratory (Brampton, Ontario) using the ThermoFisher TaqPath COVID19 Combo Kit (ThermoFisher Scientific, Waltham, MA), with
Table 1. Results of SARS-CoV-2 Detection in Paired Nasopharyngeal Swab and the Non-nasopharyngeal Swab Specimens From Individuals who Presented to an Outpatient COVID-19 Testing Center

\begin{tabular}{llcc} 
& & \multicolumn{2}{c}{ Nasopharyngeal } \\
Non-nasopharyngeal & $\begin{array}{l}\text { Non-nasopharyngeal } \\
\text { Swab }\end{array}$ & Swab Result & \multicolumn{2}{c}{\begin{tabular}{c} 
Swab \\
\cline { 3 - 4 }
\end{tabular}} & Positive & 7 & 1 \\
\hline Saline gargle & Negative & 7 & 543 \\
\hline & Positive & 44 & 1 \\
\hline Oral swab & Negative & 11 & 549 \\
\hline & Positive & 34 & 2 \\
\hline Oral-anterior nasal swab & Negative & 6 & 352 \\
\hline
\end{tabular}

detection of SARS-CoV-2 genes E, S, and ORF1ab conducted using the on real-time PCR system 7500 Fast or QuantStudio 6 (Applied Biosystems, Waltham, MA). Prior to testing, samples were inactivated by heating at $56^{\circ} \mathrm{C}$ for 30 minutes in a dry bath filled with thermal beads followed by a 30 -second vortex. Samples were extracted by combining $200 \mu \mathrm{L}$ of each patient sample with $250 \mu \mathrm{L}$ of lysis buffer master mix containing TNA lysis buffer (Omega Biotek, Norcoss, GA), Carrier RNA (Omega Bio-tek), and MS2 phage internal control (ThermoFisher). RNA was extracted using MagBind Viral RNA Xpress kit (Omega Bio-tek) on Hamilton Microlab STARlets (Hamilton, Reno, NV). A sample was defined as positive if the viral genome was detected at Ct values of $<37$ and as negative at $\mathrm{Ct}$ values $\geq 37$.

\section{Statistical analysis}

Descriptive statistics were presented as means or medians as appropriate for continuous variables and proportions for categorical variables. Indeterminate test results, in which each of the targets had Ct values $>35$, were run again and were considered positive for the purposes of analysis if both targets were detected with a $\mathrm{Ct}$ below 38 . The sensitivity and negative predictive value for nasopharyngeal and the non-nasopharyngeal tests were calculated with the reference standard being the total number of positives by each test being evaluated because no current gold standard exists. ${ }^{8,9}$ The performance characteristics were adjusted for the subsample testing strategy using inverse-probability weighting. ${ }^{10}$ The $\kappa$ coefficient was used to estimate the agreement between the nasopharyngeal swab and each self-collected technique. The difference in $\mathrm{Ct}$ values for the E-gene detected from the standard healthcare provider collected nasopharyngeal swab between the concordant and discordant pairs of each self-collected technique was analyzed using the Wilcoxon rank-sum test. All analyses were performed using $\mathrm{R}$ version 4.0.0 software (R Foundation for Statistical Computing, Vienna, Austria).

\section{Results}

Of 19,620 nasopharyngeal swabs performed in the 3 separate study periods $(14,491$ for saline gargle, 3,542 for oral swab and 1,587 for oral-anterior nose swab), 340 individuals were positive for SARSCoV-2. Of these 340, 159 (47\%) had a paired non-nasopharyngeal sample collected with $64(33 \%)$ of 194 for the saline gargle, 55 (71\%) of 77 for the oral swab, and 40 (58\%) of 69 for oral-anterior nasal swab. Overall, 608 saline gargles, 605 oral swabs, and 394 oral-anterior nasal swabs were tested. Clinical information was 
Table 2. Performance Characteristics of Nasopharyngeal and Non-nasopharyngeal Swab Detection Methods for SARS-CoV-2 by Various Reference Standards ${ }^{a}$

\begin{tabular}{|c|c|c|c|c|c|c|c|}
\hline Swab & Reference Standard & Sensitivity ${ }^{b}$ & Adjusted Sensitivity ${ }^{b}$ & Specificity ${ }^{b}$ & NPV ${ }^{b}$ & Adjusted NPV ${ }^{b}$ & $\mathrm{PPV}^{\mathrm{b}}$ \\
\hline NP & Gargle + NP & $0.98(0.92-1.00)$ & $0.88(0.83-0.92)$ & & $1.00(0.99-1.00)$ & $1.00(0.98-1.00)$ & \\
\hline Gargle & Gargle + NP & $0.89(0.79-0.96)$ & $0.90(0.86-0.94)$ & & $0.99(0.97-0.99)$ & $1.00(0.98-1.00)$ & \\
\hline Gargle & NP & $0.89(0.79-0.96)$ & $0.89(0.79-0.95)$ & $1.00(0.99-1.00)$ & $0.99(0.97-0.99)$ & $1.00(1.00-1.00)$ & $1.00(0.94-1.00)$ \\
\hline NP & Oral + NP & $0.98(0.90-1.00)$ & $0.92(0.84-0.97)$ & & $1.00(0.99-1.00)$ & $1.00(0.95-1.00)$ & \\
\hline Oral & Oral + NP & $0.80(0.68-0.90)$ & $0.82(0.72-0.89)$ & & $0.98(0.97-0.99)$ & $1.00(0.95-1.00)$ & \\
\hline Oral & NP & $0.80(0.67-0.90)$ & $0.80(0.67-0.89)$ & $1.00(0.99-1.00)$ & $0.98(0.97-0.99)$ & $1.00(1.00-1.00)$ & $0.98(0.88-1.00)$ \\
\hline NP & $\begin{array}{l}\text { Oral-Anterior } \\
\text { Nasal + NP }\end{array}$ & $0.95(0.84-0.99)$ & $0.89(0.80-0.95)$ & & $0.99(0.98-1.00)$ & $1.00(0.95-1.00)$ & \\
\hline $\begin{array}{l}\text { Oral-Anterior } \\
\text { Nasal }\end{array}$ & $\begin{array}{l}\text { Oral-Anterior } \\
\text { Nasal + NP }\end{array}$ & $0.86(0.71-0.95)$ & $0.87(0.77-0.93)$ & & $0.98(0.96-0.99)$ & $1.00(0.95-1.00)$ & \\
\hline $\begin{array}{l}\text { Oral-Anterior } \\
\text { Nasal }\end{array}$ & NP & $0.85(0.70-0.94)$ & $0.85(0.70-0.93)$ & $0.99(0.98-1.00)$ & $0.98(0.96-0.99)$ & $0.99(0.99-1.00)$ & $0.94(0.81-0.99)$ \\
\hline
\end{tabular}

Note. NP, nasopharyngeal; NPV, negative predictive value; PPV, positive predictive value.

a Unadjusted and adjusted (by inverse probability weighting) for the testing subsample were calculated.

${ }^{b}$ Data shown with $95 \%$ confidence intervals.

available for 332 (98\%) of 340 individuals positive by the nasopharyngeal swab, and $90(26 \%)$ of 340 were asymptomatic at the time of testing. The median age of individuals with COVID-19 was 33 years (IQR, 24-51), 172 (51\%) of 340 were female and $16(10 \%)$ of 159 paired specimens were obtained from children (aged $<18$ years). Those who provided a paired specimen were as likely to be asymptomatic as the group that did not provide a paired specimen (34 of 155 vs 56 of 177; $P=.06$ ) and had similar time from symptom onset to test: median, 3 (range, $1-5)$ for paired group and median 2 (range, $0-4)$ for no paired specimen group $(P=.14)$.

The saline gargle was falsely negative in 7 (11\%) of 64 samples, the oral swab in $11(20 \%)$ of 55 samples, and the oral-anterior nasal swab in $6(15 \%)$ of 40 samples (Table 1). Unadjusted, the sensitivity of the nasopharyngeal was $>90 \%$, whereas the sensitivity of the saline gargle was 0.89 (95\% confidence interval [CI], 0.79-0.96), the oral swab was 0.80 (95\% CI, 0.68-0.90), and the combined oral-anterior nasal swab 0.86 (95\% CI, $0.71-$ $0.95)$. When accounting for the random sampling of negative specimens and fraction of individuals with a paired non-nasopharyngeal swab, the sensitivity of the nasopharyngeal swab decreased and that of the non-nasopharyngeal techniques increased to 0.90 (95\% CI, 0.86-0.94) for the saline gargle, 0.82 (95\% CI, 0.72-0.89) for the oral swab, and 0.87 (95\% CI, 0.77-0.93) for the oral-anterior nasal swab (Table 2).

The percent agreement between the nasopharyngeal swab and the saline gargle was $0.99(\kappa, 0.93 ; 95 \% \mathrm{CI}, 0.86-0.96)$, for the oral swab it was $0.98(\kappa, 0.87 ; 95 \% \mathrm{CI}, 0.79-0.92)$, and for the oral-anterior nasal swab it was $0.97(\kappa, 0.85 ; 95 \% \mathrm{CI}, 0.74-0.91)$. Discordant saline gargle and oral-anterior nasal swab specimens were observed primarily in asymptomatic individuals with nasopharyngeal specimens that had high Ct values for the E-gene, whereas the oral swab was negative in both symptomatic and asymptomatic individuals (Fig. 1). The median Ct values for nasopharyngeal swab concordant and discordant saline gargle specimens were 17 and 31 $(P<.001)$, for the oral swabs these values were 17 and $28(P<.001)$ and for oral-anterior nasal swabs these values were 18 and $31(P=$ .007), respectively. The Ct values for those who tested positive in only the saline gargle were 37 for the open-reading-frame target, 33 for the E-gene target for the oral swab, and 25 and 27 for the E-gene for the oral-anterior nasal swabs.

\section{Discussion}

In outpatient COVID-19 testing centers, self-collected techniques are feasible, require minimal instruction, and preclude the presence of a healthcare professional. The combined oral-anterior nasal swab and saline gargle are comparable alternatives to a nasopharyngeal swab, but the lower sensitivity of the oral swab makes this less useful.

Saliva is effective for SARS-CoV-2 detection with an overall sensitivity of 0.91 (95\% CI, 0.80-0.99). ${ }^{4}$ The challenge with saliva has been specimen collection and processing, which has spurred the evaluation of oral swabs, which have a sensitivity of $~ 90 \%$ when a nasopharyngeal swab is the reference standard according to a small study. ${ }^{11}$ Our attempt to increase the sensitivity of oral swabs by maximizing saliva collection was unsuccessful. Using a nylon fiber swab to collect saliva missed individuals with COVID-19 who had Ct values below 24, the concentration at which SARSCoV-2 can be cultured and theoretically transmitted to others. ${ }^{12}$

Saline gargle can detect SARS-CoV-2 in symptomatic individuals with COVID-19. ${ }^{6,13}$ When evaluated in those with confirmed COVID-19, it was similar to a nasopharyngeal swab with a reported sensitivity of $98 \%{ }^{6}$ This study only tested individuals with prior SARS-CoV-2 detection and who had been symptomatic for days prior to testing. We collected paired nasopharyngeal swab and saline gargle specimens to assess the performance characteristics at the time of diagnosis, the intended use of this technique. Our study population included individuals with short durations of symptoms and those who were asymptomatic, providing a representative sample of the populations who attend COVID-19 testing centers.

Swabbing the anterior nares is less invasive than a nasopharyngeal swab and has a sensitivity of 94.0\% (97.5\% CI, 83.8-100.0), according to a small study of symptomatic individuals being tested for COVID-19. ${ }^{11}$ We found that swabbing the oropharynx and tongue along with the anterior nares had an adjusted sensitivity approaching $90 \%$, equivalent to a nasopharyngeal swab when all positive specimens were used as the reference standard. Similar to the saline gargle, the oral-anterior nasal swab missed those with high $\mathrm{Ct}$ values who were asymptomatic and less like to be infectious. ${ }^{12}$ In addition, the oral-anterior nasal swab identified 2 symptomatic individuals with $\mathrm{Ct}$ values of $\sim 25$ who were falsely negative 


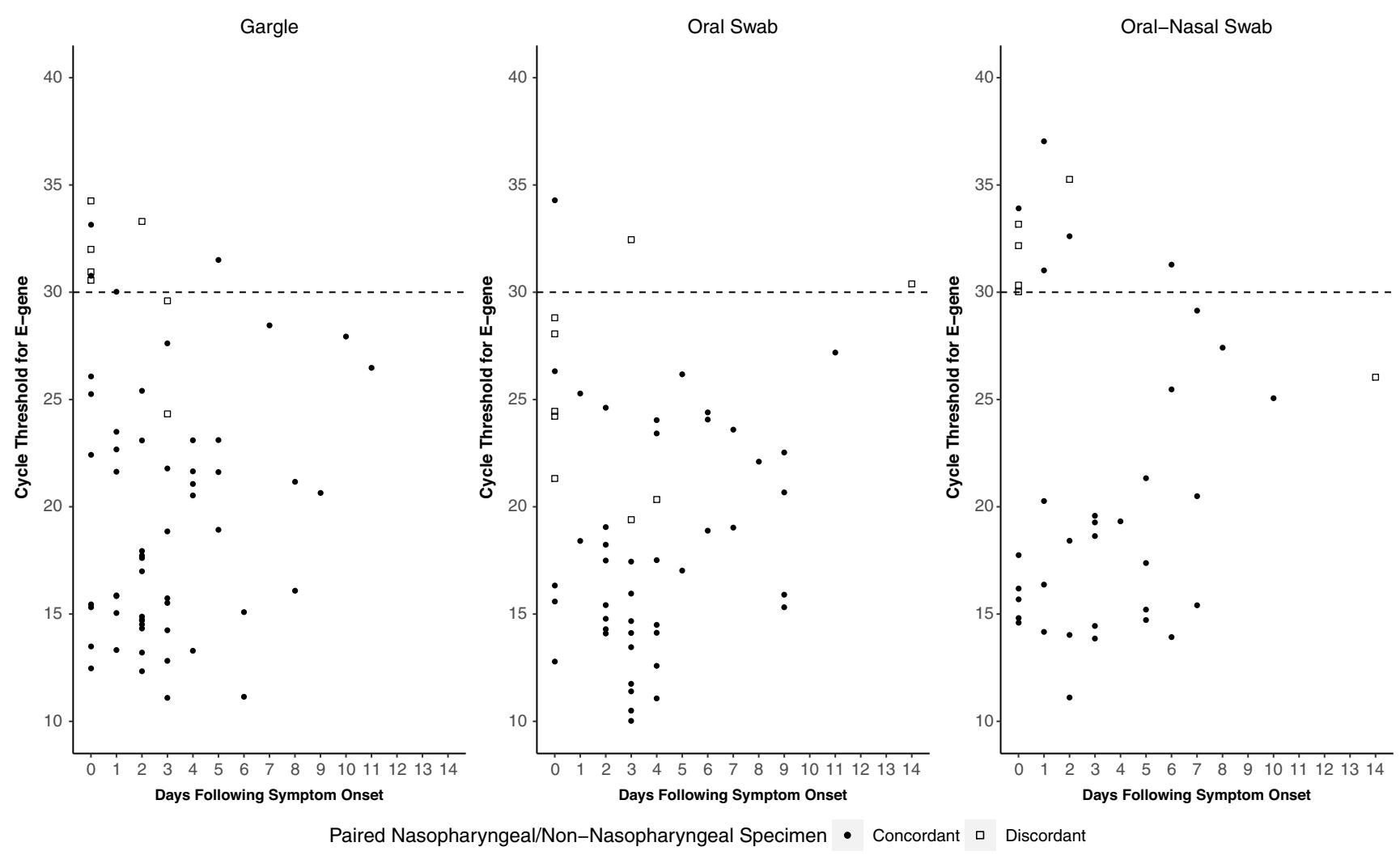

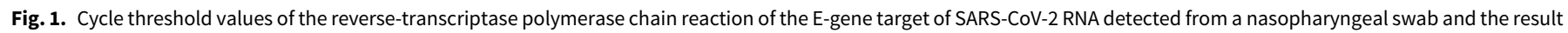
of a paired saline gargle, oral swab or combined oral-anterior nasal swab by time from symptom onset in days ( 0 indicates those asymptomatic at the time of testing).

by a nasopharyngeal swab, highlighting the fact that swabbing a second location increases the sensitivity to some degree. Added advantages of the combined oral-anterior nasal swab over the saline gargle are that these tests can be substituted for nasopharyngeal swabs in existing testing platforms, do not require proprietary collection containers, and can be transported in media that inactivates viruses, which obviates the need for heat inactivation.

This study has several limitations. First, saline gargle was tested for SARS-CoV-2 using a different platform than the nasopharyngeal swab, which can be problematic if the analytic thresholds are different. However, in a comparison of 13 different testing platforms, there was minimal difference in the detection limits of SARS$\mathrm{CoV}-2 .{ }^{14}$ Second, the proportion of individuals who provided a paired non-nasopharyngeal swab were different in each testing period. Such differences were accounted for using inverse-probability weighting, but this cannot completely overcome self-selection bias whereby individuals who submit a self-collected specimen are systematically different from those who do not. Third, the cohort consisted primarily of adults so the results may not apply to children.

In conclusion, self-collected saline gargle and a combined oralanterior nasal swab are comparable alternatives to the nasopharyngeal swab in adults presenting to outpatient COVID-19 testing centers. Both techniques are inexpensive, easy to self-collect, reduce exposure of healthcare workers and others to COVID-19, and can be operationalized using the current testing infrastructure. These options may be valuable additions to diagnostic testing, in particular in those who are mandated to undergo repeated testing.

Acknowledgments. We are grateful for the support of the clinical and clerical staff of the outpatient assessment centers. We are deeply indebted to the many individuals who provided non-nasopharyngeal samples.
Financial support. C.E.K. is supported as an Eliot Phillipson Scholar of the Faculty of Medicine, University of Toronto. This research received no specific grant from any funding agency in the public, commercial, or not-for-profit sectors.

Conflicts of interest. M.A.S., H.R., and L.W.G. are employees of Dynacare (a subsidiary of Laboratory Corporation of America), which is a private, for-profit laboratory that offers diagnostic testing services to government and nongovernment clients. Although the individuals noted herein will not personally benefit from this submission, the company could benefit financially if the test is commercially marketed. All other authors report no conflicts of interest relevant to this article.

Supplementary material. To view supplementary material for this article, please visit https://doi.org/10.1017/ice.2021.2

\section{References}

1. Kretzschmar ME, Rozhnova G, Bootsma MCJ, van Boven M, van de Wijgert JHHM, Bonten MJM. Impact of delays on effectiveness of contact tracing strategies for COVID-19: a modelling study. Lancet Pub Health 2020;5:452-459.

2. Marty FM, Chen K, Verrill KA. How to obtain a nasopharyngeal swab specimen. N Engl J Med 2020 28;382(22):e76.

3. Williamson EJ, Walker AJ, Bhaskaran $\mathrm{K}$, et al. Factors associated with COVID-19-related death using OpenSAFELY. Nature 2020;584:430-436.

4. Czumbel LM, Kiss S, Farkas N, et al. Saliva as a candidate for COVID-19 diagnostic testing: a meta-analysis. Front Med 2020;7:465.

5. Kandel C, Zheng J, McCready J, et al. Detection of SARS-CoV-2 from saliva as compared to nasopharyngeal swabs in outpatients. Viruses 2020;12(11):1314.

6. Goldfarb DM, Tilley P, Al-Rawahi GN, et al. Self-collected saline gargle samples as an alternative to healthcare-worker-collected nasopharyngeal swabs for COVID-19 diagnosis in outpatients. medRxiv 2020. doi: 10. $1101 / 2020.09 .13 .20188334$. 
7. LeBlanc JJ, Gubbay JB, Li Y, et al. Real-time PCR-based SARS-CoV-2 detection in Canadian laboratories. J Clin Virol 2020;128:104433.

8. Jamal AJ, Mozafarihashjin M, Coomes E, et al. Sensitivity of nasopharyngeal swabs and saliva for the detection of severe acute respiratory syndrome coronavirus 2 (SARS-CoV-2). Clin Infect Dis 2020. doi: 10.1093/cid/ciaa848.

9. Vandenberg O, Martiny D, Rochas O, van Belkum A, Kozlakidis Z. Considerations for diagnostic COVID-19 tests. Nat Rev Microbiol 2020. doi: 10.1038/s41579-020-00461-z.

10. Katki HA, Li Y, Edelstein DW, Castle PE. Estimating the agreement and diagnostic accuracy of two diagnostic tests when one test is conducted on only a subsample of specimens. Stat Med 2012;31:436-448.
11. Tu Y-P, Jennings R, Hart B, et al. Swabs collected by patients or health care workers for SARS-CoV-2 testing. N Engl J Med 2020 30;383: 494-496.

12. Bullard J, Dust K, Funk D, et al. Predicting infectious SARS-CoV-2 from diagnostic samples. Clin Infect Dis 2020. doi: 10.1093/cid/ciaa638.

13. Saito M, Adachi E, Yamayoshi S, et al. Gargle lavage as a safe and sensitive alternative to swab samples to diagnose COVID-19: a case report in Japan. Clin Infect Dis 2020 28;71:893-894.

14. Iglói Z, Leven M, Abou-Nouar AKZ, et al. Comparison of commercial realtime reverse transcription PCR assays for the detection of SARS-CoV-2. J Clin Virol 2020;129:104510. 